() (1) https://creativecommons.org/licenses/by/4.0/

ARTIGO

\title{
SERVIÇOS PARA ESTUDANTES COM DEFICIÊNCIA NAS UNIVERSIDADES: DIFICULDADES E DESAFIOS
}

\author{
MARIA HELENA VENÂNCIO MARTINS ${ }^{1}$ \\ ORCID: http://orcid.org/0000-0001-5964-4482 \\ FRANCISCO R. L. VIEIRA DE MELO ${ }^{2}$ \\ ORCID: http://orcid.org/0000-0001-5902-9615 \\ CÁTIA MARTINS ${ }^{3}$ \\ ORCID: https://orcid.org/0000-0002-1819-8516
}

\begin{abstract}
RESUMO: Os estudantes com deficiência podem enfrentar inúmeros obstáculos e desafios ao acessar o Ensino Superior (ES) e tentar ter sucesso nele. Os Serviços de Apoio para Estudantes com Deficiência nas universidades desempenham um papel importante no fornecimento de apoio direto e indireto para esses estudantes. Este artigo apresenta as perspectivas dos Coordenadores dos Serviços de Apoio no que diz respeito à prestação de serviços a estudantes com esta condição, descrevendo as suas características, dificuldades e desafios. Os dados foram coletados em 62 instituições universitárias públicas do Brasil e de Portugal por meio de questionário desenvolvido para esse fim. Apesar das demandas enfrentadas pelos Serviços de Apoio para Estudantes com Deficiência, os sinais de bom progresso no apoio a estes estudantes estão aumentando. Embora os serviços desempenhem um papel importante, uma autonomia mínima e dificuldades significativas permanecem em vários domínios de gestão e intervenção. São discutidas as mudanças e adaptações necessárias no apoio a estes estudantes nas universidades, considerando as consequências significativas da inclusão e do sucesso para os estudantes com deficiência. Políticas inclusivas baseadas na equidade e igualdade de oportunidades e mais recursos são necessários para garantir os direitos de todos os estudantes no Ensino Superior.
\end{abstract}

Palavras-chave: estudantes com deficiência, educação inclusiva, serviços para estudantes com deficiência, ensino superior.

\footnotetext{
${ }^{1}$ Professora Assistente (UAlg). Faro, Portugal. mhmartin@ualg.pt

2 Professora Assistente (UFRN). Natal, RN, Brazil. ricardolins67@gmail.com

${ }_{3}^{3}$ Professora Assistente (UAlg). Faro, Portugal. csmartins@ualg.pt 


\title{
SERVICES FOR STUDENTS WITH DISABILITIES AT UNIVERSITIES: DIFICULTIES AND CHALLENGES
}

\begin{abstract}
Students with disabilities may confront numerous obstacles and challenges when reaching Higher Education (HE) and attempting to be successful in it. Disability Services Offices at universities play an important role in providing direct and indirect support for these students. This paper presents the perspectives of Disability Services Offices' staff regarding the provision of services to students with disabilities, describing the characteristics, difficulties and challenges of these offices. We collected data at 62 public university organizations in Brazil and Portugal using a questionnaire developed for this purpose. Despite the demands faced by the Disability Services Offices, signs of good progress in support for students with disabilities are increasing. Although the services play an important role, minimal autonomy and significant difficulties remain in several domains of management and intervention. We discuss necessary changes and adaptations in supporting these students at universities, considering the significant consequences of integration and success for students with disabilities. Inclusive policies that are based on equity and equal opportunities and more resources are required to guarantee the rights of all students in Higher Education.
\end{abstract}

Keywords: students with disabilities, inclusive education, disability services offices, higher education.

\section{SERVICIOS PARA ESTUDIANTES CON DISCAPACIDAD EN LAS UNIVERSIDADES: DIFICULTADES Y DESAFÍOS}

RESÚMEN: Los estudiantes con discapacidades pueden enfrentar muchos obstáculos y desafíos cuando acceden a la educación superior (ES) e intentan tener éxito en ella. Los Servicios de Apoyo para Estudiantes con Discapacidades en las universidades juegan un papel importante al proporcionar apoyo directo e indirecto a estos estudiantes. Este documento presenta las perspectivas del personal de los Servicios de Apoyo con respecto a la prestación de servicios a estudiantes con discapacidad, describiendo sus características, dificultades y desafíos. Los datos fueron recolectados en 62 organizaciones universitarias públicas en Brasil y Portugal utilizando un cuestionario desarrollado para este propósito. Apesar de las demandas que enfrentan los Servicios de Apoyo para Estudiantes con Discapacidad, están aumentando las señales de buen progreso en el apoyo a los estudiantes. Aunque los servicios desempeñan un papel importante, subsisten una autonomía mínima y dificultades significativas en varios dominios de gestión e intervención. Se discuten los cambios y las adaptaciones necesarias en el apoyo a estudiantes en las universidades, considerando las consecuencias significativas de la inclusión y el éxito para los estudiantes con discapacidad. Se requieren políticas inclusivas basadas en la equidad y la igualdad de oportunidades y más recursos para garantizar los derechos de todos los estudiantes de Educación Superior.

Palabras clave: estudiantes con discapacidad, educación inclusiva, oficinas de servicios para discapacitados, educación superior.

\section{INTRODUÇÃO}

De acordo com as Nações Unidas (2015), estima-se que 1 bilhão de pessoas no mundo vivam com uma ou mais deficiências físicas, sensoriais, intelectuais ou mentais. As pessoas com deficiência são a maior e mais desfavorecida minoria do mundo. Diversos regulamentos têm orientado a garantia efetiva dos direitos das pessoas, em particular a Convenção sobre os Direitos das Pessoas com Deficiência (CDPD) e o seu Protocolo Opcional (UNITED NATIONS, 2006). Segundo a CDPD, a deficiência é definida através de um modelo ecológico, no qual a deficiência é percebida como um conceito em evolução que reflete a interação entre as atitudes individuais e sociais e o ambiente físico, económico e político que impedem a participação plena e igualitária das pessoas com deficiência na sociedade. 
Uma das principais preocupações tem sido o aumento do acesso de estudantes com deficiência ao Ensino Superior (ES) (MAJOKO; DUNN, 2018; WILLIAMS, POLLARD, TAKALA, HOUGHTON, 2019). Esta crecente diversidade produziu uma maior consciência da necessidade de adaptações no ES, gerando novos recursos institucionais e respostas (COSTA; PIECZKOWSKI, 2020; MORIÑA; MORGADO; LÓPEZ, 2017; NOLAN; GLEESON; TREANOR; MADIGAN, 2015).

Diversas perspectivas têm norteado o apoio aos estudantes com deficiência nas últimas décadas (LIPKA; BARUCH; MEER, 2019), e têm influenciado o desenvolvimento de políticas e disposições legais (VAN MIEGHEM ET AL., 2020). Atualmente, o modelo social postula que a deficiência é causada pela forma como a sociedade está organizada, e que as diferenças são resultados diretos das barreiras sociais, ambientais e atitudinais. Este modelo afirma que as barreiras incapacitantes devem seguir um processo de desmantelamento nas sociedades, com foco na lacuna existente entre os direitos dos estudantes e as práticas porque, como Titchkosky (2008) afirmou, a deficiência parece estar em toda parte, mas em lugar nenhum. Além disso, de acordo com o Education 2030 Framework for Action, "é necessário abordar todas as formas de exclusão e marginalização" (UNESCO, 2017, p. 12). O modelo social da deficiência é amplamente aceite como a forma mais eficaz pela qual as universidades podem responder às necessidades dos estudantes com deficiência. De acordo com este modelo, a resposta à deficiência não tem a ver com "remediar" os indivíduos, mas sim com a reestruturação dos ambientes e atitudes ao seu redor (TITCHKOSKY, 2008).

Costa e Pieczkowski (2020) apontam que há uma procura crescente do acesso de estudantes com deficiência às instituições de ensino superior. Como resultado desse aumento, o número de universidades que oferecem serviços de apoio para estudantes com deficiência cresceu consideravelmente na última década em todo o mundo. No entanto, nem todos os estdantes com deficiência recebem um adequado e igual nível de apoio, permitindo-lhes ter igualdade de oportunidades e sucesso académico (MORIÑA, 2017). De acordo com Laya (2020), a dimensão pedagógica da equidade no ES é muito importante para superar as desigualdades persistentes, apesar das políticas adotadas em todo o mundo para expandir as oportunidades educacionais.

Embora exista uma vasta diversidade de denominações para estes serviços, muitas universidades designam-nos por Serviços de Apoio para Estudantes com Deficiência (Disability Services Offices - DSOs), conceito que será usado nesta pesquisa.

A missão destes serviços é promover o compromisso das universidades com a equidade e a diversidade, por meio da prestação de serviços e acomodações académicas aos estudantes com deficiência. Além disso, estes serviços devem fornecer aconselhamento e assistência individual aos estudantes, desde a sua entrada no ES até à sua formatura, e prepará-los para a transição para o mercado de trabalho (MAJOKO; DUNN, 2018; MORIÑA, 2017; MORGADO; LÓPEZ-GAVIRA, 2017).

Uma das principais preocupações dos Serviços de Apoio é identificar as necessidades e a satisfação dos alunos e, ao mesmo tempo, promover elevados padrões académicos (DATTA; TALUKDAR, 2017). Apesar de alguns estudos nesta área, permanece a necessidade de pesquisas mais abrangentes sobre estes serviços nas universidades (HOUSE OF COMMONS LIBRARY, 2020). A maioria das investigações sobre as experiências dos alunos é limitada a uma ou apenas algumas universidades de um país, e os estudos comparando países ou continentes são escassos; assim, focamos nas experiências de dois países: Brasil e Portugal. A seleção destes dois países é baseada num passado e em raízes comuns e na partilha da mesma língua.

\section{SERVIÇOS DE APOIO NAS UNIVERSIDADES}

O acesso ao ES para pessoas com deficiência apresenta oportunidades e também desafios. Embora os governos tendam a incorporar princípios como justiça social, igualdade de oportunidades, acessibilidade e design universal (ACKAH-JNR; DANSO, 2019; STIEFEL; SHIFERAW; SCHWARTZ; GOTTFRIED, 2018) nas políticas públicas, estes desenvolvimentos não têm sido nem rápidos nem fáceis.

Alguns pesquisadores têm-se concentrado neste desafio e têm vindo a estudar este 'dever', demonstrando que os esforços das universidades para garantir oportunidades iguais aos estudantes com 
deficiência permanecem insuficientes; persiste uma lacuna entre o discurso político e a realidade prática (DATTA; TALUKDAR, 2017; WILLIAMS; POLLARD; TAKALA; HOUGHTON, 2019).

A implementação de Serviços de Apoio nas universidades foi uma contribuição importante para a inclusão de estudantes com deficiência no ES. Embora as funções destes serviços possam diferir entre os países, geralmente estes recebem e dão as boas-vindas a alunos, professores, funcionários e visitantes com deficiência e garantem um ambiente de trabalho e aprendizagem acessível e amigável. De acordo com a United Nations Educational Scientific and Cultural Organization e a World Conference on Higher Education (Paris, 5-9 October 1998), estes serviços devem fornecer assistência adequada e oportunidades para os estudantes com deficiência, para que estes possam competir em pé de igualdade com os seus colegas. Os Serviços de Apoio devem ajudar os estudantes a acessar todos os programas, serviços e atividades patrocinados pela universidade, aconselhar e apoiar os estudantes no acesso às salas de aula e outras acomodações. Finalmente, os serviços devem ser um ponto de referência no campus, garantindo os recursos adequados e auxiliando os estudantes nas suas transições ambientais, de casa para a universidade e da universidade para o trabalho, promovendo a sua autonomia e permitindo-lhes desenvolver estratégias ao longo da vida para a gestão independente das suas deficiências (UNESCO, 2017).

Apesar desta importância, a investigação de Lipka, Baruch e Meer (2019) aponta que embora muitos docentes $(58 \%)$ relatem conhecer os serviços de apoio na universidade, ainda há um desconhecimento sobre esses serviços (34\%).

Alguns estudos têm focalizado os recursos destes serviços, como a sua composição e sistemas de monitorização. Outros têm destacado as respostas diretas dos serviços, como as acomodações, divulgações, documentação e outros aspetos; enquanto outros enfatizaram as experiências de universidades isoladas ou de várias universidades num país (ACKAH-JNR; DANSO, 2019; MESSIOU, 2019; STIEFEL ET AL., 2018).

\section{POLÍTICA DE ENSINO SUPERIOR E EDUCAÇÃO INCLUSIVA NO BRASIL E EM PORTUGAL}

Em linha com as diretrizes internacionais, os dois países assinaram a Convenção sobre os Direitos das Pessoas com Deficiência (UNITED NATIONS, 2006), dando uma maior atenção à inclusão de estudantes com deficiência no ES.

Desde 2003, o Brasil vem desenvolvendo medidas legislativas para criar respostas adequadas às necessidades dos estudantes com deficiência que ingressam no ES. Embora em Portugal não exista legislação específica, como Estado membro da União Europeia, Portugal tem seguido as orientações para garantir o direito à educação das pessoas com deficiência. Na ausência de legislação geral, as universidades portuguesas têm vindo a desenvolver regulamentos específicos para proteger e promover os direitos dos estudantes com deficiência. Uma das principais consequências foi a fundação do Grupo de Trabalho para apoiar Estudantes com Deficiência no Ensino Superior (GTAEDES). Este grupo de trabalho fornece serviços, promove intercâmbios entre agências e experiências e desenvolve fundamentos para iniciativas e recursos conjuntos (MELO; MARTINS, 2016).

Segundo Melo e Martins (2016), embora a maioria das instituições de ambos os países ofereça atendimento a estudantes com deficiência, persistem dificuldades significativas na inclusão destes estudantes. A existência de normas legais no Brasil e o desenvolvimento de estatutos e regulamentos nas instituições portuguesas ainda não permitiram que os sistemas educativos sejam considerados verdadeiramente inclusivos.

Existem políticas e legislações que garantem o acesso e a permanência dos estudantes com deficiência no ES brasileiro e um orçamento para ações que promovam e garantam o acesso desses estudantes nas universidades públicas federais; no entanto, muitas universidades ainda não colocaram em prática o que é exigido por lei. De facto, e apesar dos inquestionáveis avanços das políticas inclusivas no ES brasileiro, as diretrizes pedagógicas para o estudante com deficiência ainda são incipientes em comparação ao que se estabelece para a educação básica, na qual existe uma estrutura mais ampla de serviços e recursos à disposição dos alunos (MELO; MARTINS, 2016). Situação semelhante ocorre em 
Portugal, em que os estudantes com deficiência encontram vários obstáculos no ES (MARTINS ET AL., 2015).

Considerando o que foi apresentado, questionamos: como estão organizados estes Serviços de Apoio para os estudantes com deficiência nas universidades públicas do Brasil e de Portugal? Quem são os profissionais que garantem este apoio? Quem são os estudantes que se candidatam a estes serviços? Como está estruturado o apoio e quais os recursos que estes serviços possuem? Quais são as dificuldades e desafios?

Os objetivos do presente estudo visam caracterizar (1) os Serviços de Apoio a estes estudantes nas universidades públicas do Brasil e de Portugal, (2) o funcionamento destes serviços e (3) as suas características de coordenação. As dificuldades e desafios são ainda analisados de forma a propor diretrizes globais que possam melhorar a sua eficácia e contribuir para a inclusão e o sucesso destes estudantes.

\section{METODOLOGIA}

Esta pesquisa foi realizada no contexto de um projeto envolvendo dois parceiros, Brasil e Portugal. O estudo é exploratório, transversal e comparativo, utilizando a análise quantitativa de dados através de uma pesquisa online, de forma a alcançar um número mais amplo de profissionais numa área geográfica maior.

\section{Participantes e procedimentos}

Participaram no estudo profissionais de Serviços de Apoio brasileiros e portugueses de universidades públicas financiadas pelo governo central. O critério de inclusão no estudo consistiu em ter um serviço de apoio para atender estes estudantes.

Após o desenvolvimento e adaptação de um questionário nos dois países sobre a existência de Serviços de Apoio em instituições públicas de ES, foi enviado um e-mail para todas as universidades públicas que atendiam aos critérios definidos. O e-mail foi enviado ao coordenador ou responsável de cada Serviço de Apoio solicitando a sua participação na pesquisa online.

Os participantes foram informados sobre o objetivo do estudo, o anonimato e sigilo das respostas.

Participaram dois grupos de coordenadores de Serviços de Apoio: o primeiro composto por 54 profissionais brasileiros e o segundo por 8 coordenadores portugueses. Ambas as amostras refletem a maioria dos serviços públicos dos dois países, apesar do número de respostas portuguesas ser relativamente pequeno (representando $87 \%$ dos serviços no Brasil e $75 \%$ dos Serviços de Apoio em Portugal).

\section{Instrumentos, recolha de dados, e análise de resultados}

Foram seguidas quatro etapas diferentes: (1) construção da pesquisa preliminar, (2) avaliação por juízes/avaliadores externos (pesquisadores da área de Inclusão no Ensino Superior), (3) teste piloto e (4) adaptações de linguagem.

Para o desenvolvimento da pesquisa preliminar, utilizamos como referência a literatura sobre educação e políticas inclusivas (e.g., FOTIM, 2011). Três especialistas (ou seja, dois professores de educação especial e um psicólogo) foram convidados a avaliar o conteúdo final das pesquisas (por exemplo, gramática, sintaxe e locais). A versão final foi aplicada em duas universidades brasileiras (teste piloto).

Embora ambos os países falem português, foi aplicado um teste piloto em Portugal com dois especialistas em educação especial, e as sugestões, relativas ao português europeu, também foram consideradas.

Três assuntos principais foram abordados: (1) organização dos Serviços de Apoio e características do serviço e da universidade (e.g., nome, recursos financeiros e acessibilidade); (2) 
funcionamento do Serviço de Apoio (e.g., equipa, processo de seleção, serviços de consultoria, formação, projetos e parcerias); e (3) características da coordenação (e.g., género, idade, formação académica, experiência e percepções sobre o serviço). A pesquisa incluiu uma mistura de questões de escolha fechada e forçada (20 questões), escalas Likert (44 itens) e oportunidades para comentários.

As pesquisas foram enviadas por e-mail para as universidades portuguesas e brasileiras que possuíam Serviços de Apoio (critério de inclusão da pesquisa).

De forma a garantir os princípios éticos, os pesquisadores informaram todos os participantes sobre os objetivos e relevância do estudo. Os participantes forneceram o seu consentimento informado para participar no estudo. Foi respeitado o princípio da confidencialidade das informações, garantido aos participantes. Todos os documentos relativos aos participantes foram mantidos em pasta própria em local seguro. O direito de desistência do estudo foi garantido sem restrições aos participantes. Foram ainda tranquilizados de que não seriam afetados pelas suas declarações e que todas as suas observações permaneceriam confidenciais.

Os dados quantitativos foram importados para o Statistical Package for the Social Sciences V. 26 (IBM SPSS) e foi realizada uma apresentação analítica usando estatísticas descritivas (e.g., médias, desvio padrão, intervalos e percentagens).

\section{RESULTADOS}

\section{Organização dos Serviços de Apoio}

No que diz respeito às características dos Serviços de Apoio, é importante referir que estes surgiram no final da década de 1980 em Portugal e, no Brasil, surgiram quase uma década depois (1999). Os anos médios de existência dos serviços refletem essas diferenças (Brasil: Max $=17, M=5.67, S D=$ 3.19; Portugal: $\operatorname{Max}=28, M=9.63, S D=9.62$ ). Ambos os países indicaram regulamentações específicas para o apoio e funcionamento de seus serviços (Brasil $=61.10 \%$, Portugal $=87.50 \%$ ).

Em relação à organização dos serviços, nenhum relatou ser totalmente autónomo. Em geral, nos dois países, os serviços estão dependentes das Faculdades (Brasil: 72.8\%) e dos Serviços da Reitoria /Reitoria (Portugal: 37.5\%). Os resultados revelaram baixos níveis de autonomia para decidir e desenvolver projetos. Outra condição importante era ter orçamento específico: no Brasil, a maioria dos serviços (55.6\%) tem orçamento próprio; em Portugal, a maioria (75\%) não dispõe de recursos financeiros específicos afetados.

No que diz respeito à acessibilidade das instalações, os coordenadores portugueses mostraram-se mais otimistas quanto à oferta de serviços $(\mathrm{Sim}=62.5 \%)$ do que os coordenadores brasileiros $($ Parcial $=68.5 \%)$. Quanto à acessibilidade interna, as opiniões foram positivas e concordantes, evidenciando a existência de condições de acessibilidade nas universidades dos dois países. No entanto, é de referir que alguns inquiridos continuaram a referir dificuldades de acessibilidade (Brasil, Parcial $=$ 44,4\%; Portugal, Parcial $=25 \%$ ). Uma minoria relatou que os estudantes com deficiência não tinham acomodações suficientes com acessibilidade (e.g., rampas de acesso, elevadores, salas de aula adaptadas; Brasil, Não $=7.4 \%$; Portugal, Não $=12.5 \%$.

\section{Dados demográficos do staff dos Serviços de Apoio}

A maioria dos coordenadores dos Serviços de Apoio são do género feminino (Brasil = $83.3 \%$; Portugal $=87.5 \%$ ), e alguns coordenadores apresentavam alguma deficiência (Brasil $=13 \%$; Portugal $=12.5 \%$ ). Quanto às habilitações literárias, a maioria tem doutoramento (Brasil $=51.9 \%$; Portugal $=75 \%)$, principalmente em Ciências Sociais $($ Brasil $=64.8 \%$; Portugal $=75 \%)$ e Humanidades $($ Brasil $=14.8 \%$; Portugal $=25 \%)$. Possuíam especializações na área da Educação Especial (Brasil $=$ $79.6 \%$; Portugal $=50 \%$ ), vasta experiência docente no ES (Brasil, $M=19.06$ anos; Portugal, $M=21.6$ anos) e experiência como elementos dos Serviços de Apoio (Brasil, $M=8.44$ anos; Portugal, $\mathrm{M}=6.28$ anos) (Tabela 1). 
Tabela 1- Características do perfil dos Coordenadores

\begin{tabular}{|c|c|c|c|c|}
\hline \multirow{2}{*}{ Características } & \multicolumn{2}{|c|}{ Brazil $(n=54)$} & \multicolumn{2}{|c|}{ Portugal $(n=8)$} \\
\hline & $n$ & $\%$ & $n$ & $\%$ \\
\hline Masculino & 9 & 16.7 & 1 & 12.5 \\
\hline Feminino & 45 & 83.3 & 7 & 87.5 \\
\hline Condição de deficiência & 7 & 13 & 1 & 12.5 \\
\hline Especialização & 10 & 18.5 & 2 & 25 \\
\hline Mestrado & 16 & 29.6 & 0 & 0 \\
\hline Doutoramento (PhD) & 28 & 51.9 & 6 & 75 \\
\hline Formação Especializada & 43 & 79.6 & 4 & 50 \\
\hline $\begin{array}{l}\text { Experiência com } \\
\text { Deficiências }\end{array}$ & 43 & 79.6 & 7 & 87.5 \\
\hline \multirow[t]{2}{*}{ Experiência de gestão } & 47 & 87 & 6 & 75 \\
\hline & Min (Max) & $M(D P)$ & $\operatorname{Min}(\operatorname{Max})$ & $M(D P)$ \\
\hline $\begin{array}{l}\text { Anos de trabalho na } \\
\text { Universidade }\end{array}$ & $1(30)$ & $19.06(10.19)$ & $8(29)$ & $21.63(6.74)$ \\
\hline $\begin{array}{l}\text { Anos de serviço na } \\
\text { Coordenação }\end{array}$ & $0.1(9.8)$ & $8.44(7.70)$ & $0.25(18)$ & $6.28(5.76)$ \\
\hline
\end{tabular}

Notas: Min (Minimo), Max (Máximo), $M$ (Média), DP (Desvio Padrão).

Fonte: Tabela elaborada pelos autores a partir dos dados extraídos da investigação efetuada.

Globalmente, os Serviços de Apoio nas universidades brasileiras empregam equipas maiores e mais diversificadas $(M=6, D P=5.29$, Min = 1, Max =24) do que os Serviços de Apoio portugueses $(M=3.43, D P=2.37, \operatorname{Min}=1, \operatorname{Max}=8)($ Tabela 2$)$.

Tabela 2 - Professionais dos Serviços de Apoio

\begin{tabular}{lcccccc}
\hline \multirow{2}{*}{ Formação Específica } & \multicolumn{3}{c}{ Brazil $(\boldsymbol{n}=\mathbf{5 4 )}$} & \multicolumn{3}{c}{ Portugal $(\boldsymbol{n}=\mathbf{8})$} \\
& $\mathbf{0}$ & $\boldsymbol{M}$ & $\boldsymbol{D P}$ & $\mathbf{\%}$ & $\boldsymbol{M}$ & $\boldsymbol{D P}$ \\
\hline Professor de Educação Especial & 46.3 & 0.77 & 1.00 & 37.5 & 0.88 & 0.99 \\
Psicopedagogo & 77.8 & 0.25 & 0.65 & 87.5 & 0 & 0 \\
Intérprete de Língua Gestual & 24.1 & 2.77 & 2.94 & 75.0 & 0.14 & 0.38 \\
Tradutor & 94.4 & 0.08 & 0.56 & 87.5 & 0 & 0 \\
Revisor de Braille & 79.6 & 0.25 & 0.62 & 75.0 & 0.38 & 0.74 \\
Psicólogo & 63.0 & 0.58 & 1.04 & 25.0 & 1 & 0.82 \\
Assistente Social & 66.7 & 0.71 & 1.70 & 50.0 & 0.57 & 0.79 \\
\hline & $\boldsymbol{M}$ & $\boldsymbol{D P}$ & Min (Max) & $\boldsymbol{M}$ & $\boldsymbol{S D}$ & Min (Max) \\
\hline Staff & 6 & 5.29 & $1(24)$ & 3.43 & 2.37 & $1(8)$ \\
\hline
\end{tabular}

Notas: Min (Minimo), Max (Máximo), $M$ (Média), DP (Desvio Padrão).

Fonte: Tabela elaborada pelos autores a partir dos dados extraídos da investigação efetuada.

Em relação aos recursos de educação especial, os participantes referem intérpretes de Língua Gestual (Brasil: $M=2.77, D P=2.94$; Portugal: $M=0.14, D P=0.34$ ), professores de educação especial (Brasil: $M=0.77, D P=1.00$; Portugal: $M=0.88, D P=0.99$ ) e psicólogos (Brasil: $M=0.58, D P=1.04$; Portugal: $M=1.00, D P=0,82$ ) como os profissionais mais comuns que trabalham nos Serviços de Apoio. No Brasil, também são referidos psicopedagogos a trabalhar nos serviços $(M=0.25, D P=0.65)$ (Tabela 2). 


\section{Perfil dos estudantes com Deficiêcia que são acompanhados nos Serviços de Apoio}

O perfil dos estudantes que são acompanhados pelos serviços apresentaram algumas semelhanças. Em ambos os países, as deficiências mais frequentes foram as deficiências físicas (Brasil, $M$ $=21.01, D P=34.66$; Portugal, $M=5.62, D P=5.73$ ), a deficiência visual (Brasil, $M=12.47, D P=35.32$; Portugal, $M=1.87, D P=2.97$ ), a deficiência auditiva (Brasil, $M=9.77, D P=16.04$; Portugal, $M=1.25$, $D P=1.36$ ), deficiências múltiplas (Brasil, $M=2.91, D P=5.58$; Portugal, $M=0.25, D P=0.53$ ) e deficiências cognitivas (Brasil, $M=1.54, D P=3.56$; Portugal, $M=0.12, D P=0,23$ ). Em Portugal, foram também designados outros problemas, como as doenças psiquiátricas $(M=4.5, D P=6.46)$, dislexia $(M$ $=4, D P=5.35)$, doenças crónicas $(M=2.38, D P=4.07)$ e doenças neurológicas $(M=0.75, S D=1.75)$.

\section{Funcionamento dos Serviços de Apoio}

De maneira geral, os participantes brasileiros expressaram uma apreciação mais positiva do funcionamento dos Serviços de Apoio, considerando a assessoria pedagógica a professores e diretores sobre acessibilidade e inclusão (90.7\%), desenvolvimento de projetos de ensino (monitorização, tutoria e voluntariado) para apoio académico a estudantes com deficiência (85.2\%), disponibilização de recursos de tecnologia assistida (83.3\%), formação contínua para docentes e não-docentes em acessibilidades e inclusão (81.5\%) e desenvolvimento de projetos de extensão (parcerias extra-institucionais para o desenvolvimento da inclusão) $(81.5 \%$ ) Em relação aos serviços prestados com menor frequência, os coordenadores brasileiros mencionaram domínios relacionados com a atenção especializada a estudantes com deficiência $(57.4 \%)$.

Os inquiridos portugueses apontaram como os empreendimentos mais bem sucedidos o desenvolvimento de projetos de aprendizagem (acompanhamento, tutoria e voluntariado) para apoio académico a estudantes com deficiência (87.5\%), desenvolvimento de projetos de investigação (87.5\%), assessoria pedagógica a docentes e directores sobre acessibilidade e inclusão $(62.5 \%)$ e apoio especializado aos estudantes com deficiência (62.5\%). Os serviços prestados com menor frequência pelos Serviços de Apoio portugueses relacionam-se com as parcerias comunitárias (25\%) (Tabela 3).

Tabela 3 - Domínios de apoio e parcerias promovidas pelos Serviços de Apoio

\begin{tabular}{lcccccccc}
\hline & \multicolumn{3}{c}{ Brazil $(\boldsymbol{n}=\mathbf{5 4})$} & \multicolumn{3}{c}{ Portugal $(\boldsymbol{n}=\mathbf{8})$} \\
\cline { 2 - 9 } Domínios de apoio & \multicolumn{2}{c}{ Não } & \multicolumn{2}{c}{ Sim } & \multicolumn{3}{c}{ Não } & \multicolumn{2}{c}{ Sim } \\
\cline { 2 - 9 } & $\boldsymbol{n}$ & $\mathbf{0}$ & $\boldsymbol{n}$ & $\mathbf{0}$ & $\boldsymbol{n}$ & $\mathbf{\%}$ & $\boldsymbol{n}$ & $\mathbf{\%}$ \\
\hline Processos de Seleção & 12 & 22.2 & 41 & 75.9 & 5 & 62.5 & 3 & 37.5 \\
Acompanhamento Pedagógico & 5 & 9.3 & 49 & 90.7 & 3 & 37.5 & 5 & 62.5 \\
Formação Contínua & 10 & 18.5 & 44 & 81.5 & 4 & 50 & 4 & 50 \\
Orientações Tecnológicas Assistidas & 10 & 18.5 & 43 & 79.6 & 4 & 50 & 4 & 50 \\
Acesso Tecnológico Assitido & 9 & 16.7 & 45 & 83.3 & 4 & 50 & 4 & 50 \\
Produção de Material Acessível & 16 & 29.6 & 38 & 70.4 & 2 & 25 & 6 & 75 \\
Atendimento Especializado & 22 & 40.7 & 31 & 57.4 & 3 & 37.5 & 5 & 62.5 \\
Ensino de Desenvolvimento de Projetos & 8 & 14.8 & 46 & 85.2 & 1 & 12.5 & 7 & 87.5 \\
Desenvolvimento de Projetos de Pesquisa & 11 & 20.4 & 43 & 79.6 & 1 & 12.5 & 7 & 87.5 \\
Desenvolvimento de Projetos de Extensão & 9 & 16.7 & 44 & 81.5 & 6 & 75 & 2 & 25 \\
\hline
\end{tabular}

Fonte: Tabela elaborado pelos autores a partir dos dados extraídos da investigação efetuada. 
Os Serviços de Apoio de ambos os países afirmaram estar envolvidos em parcerias consistentes, embora os profissionais do Brasil assinalem a cooperação interna (e.g., entre faculdades e institutos universitários; 85.2\%) e os portugueses assinalem mais a cooperação externa (e.g., associações, escolas secundárias, serviços do governo local; 75\%).

Em geral, têm vindo a ser feitos esforços para garantir que os apoios prestados pelos Serviços sejam adequados às necessidades dos estudantes. Os dados revelaram que os Serviços de Apoio brasileiros parecem estar melhor equipados para incluir estudantes do que os Serviços de Apoio portugueses. Globalmente, observou-se a falta de vários recursos (Brasil: guia tradutor, 90.7\%; revisor Braille, 75.9\%; transporte acessível, 54.7\%; Portugual: guia tradutor, 87.5\%; revisor Braille, 75\%; e transporte acessível, 100\%). Em relação aos recursos insuficientes, os respondentes brasileiros apontaram vários como insatisfatórios (recursos tecnológicos, 69.8\%; intérprete de língua gestual, 55.6\%; e recursos de áudio, 53.7\%). Os coordenadores dos Serviços de Apoio portugueses indicaram mobiliário acessível (62.5\%) e os recursos didáticos digitais (37.5\%) como insuficientes. Os participantes consideraram vários tipos de recursos suficientes (Brasil: intérprete de língua gestual 31.5\%; recursos em braile, 31.5\%; materiais educacionais acessíveis, 31.5\%; Portugal: recursos tecnológicos, $75 \%$; recursos didáticos, $62.5 \%$; e recursos de áudio, $50 \%$ ).

Em relação às dificuldades percebidas pelos coordenadores (Tabela 4), ambos os grupos concordaram com várias preocupações em relação às condições e recursos dos Serviços de Apoio. Os profissionais brasileiros referem a falta de profissionais suficientes $(87 \%)$, professores especializados $(66.7 \%)$ e apoio dos professores $(62.3 \%)$ como as áreas mais problemáticas.

Tabela 4 - Perceções dos Professionals relativamente às Dificuldades nos Serviços de Apoio

\begin{tabular}{|c|c|c|c|c|c|c|c|c|}
\hline \multirow{3}{*}{ Dificuldades Percebidas } & \multicolumn{4}{|c|}{ Brazil $(n=54)$} & \multicolumn{4}{|c|}{ Portugal $(n=8)$} \\
\hline & \multicolumn{2}{|c|}{ Não } & \multicolumn{2}{|c|}{ Sim } & \multicolumn{2}{|c|}{ Não } & \multicolumn{2}{|c|}{ Sim } \\
\hline & $n$ & $\%$ & $n$ & $\%$ & $n$ & $\%$ & $n$ & $\%$ \\
\hline Condições de trabalho & 25 & 46.3 & 28 & 51.9 & 4 & 50 & 4 & 50 \\
\hline Professionais Insuficientes & 6 & 11.1 & 47 & 87 & 5 & 62.5 & 3 & 37.5 \\
\hline Formação sobre Inclusão para prpfessores & 17 & 31.5 & 36 & 66.7 & 0 & 0 & 8 & 100 \\
\hline Recursos Financeiros Insuficientes & 27 & 50.9 & 26 & 49.1 & 5 & 62.5 & 3 & 37.5 \\
\hline Não Envolvimento dos Estudantes & 39 & 73.6 & 14 & 26.4 & 5 & 62.5 & 3 & 37.5 \\
\hline Falta de Apoio aos Professores & 20 & 37.7 & 33 & 62.3 & 6 & 75 & 2 & 25 \\
\hline Insuficiente Apoio Institutional & 44 & 83 & 9 & 17 & 8 & 100 & 0 & 0 \\
\hline Dificuldades nas Parcerias & 44 & 83 & 9 & 17 & 5 & 62.5 & 3 & 37.5 \\
\hline Recursos de Tecnologia Assistida & 25 & 47.2 & 28 & 52.8 & 5 & 62.5 & 3 & 37.5 \\
\hline Dificuldades nas Interações Professionais & 50 & 94.3 & 3 & 5.7 & 8 & 100 & 0 & 0 \\
\hline
\end{tabular}

Fonte: Tabela elaborada pelos autores a partir dos dados extraídos da investigação efetuada.

Os profissionais portugueses consideraram como domínios insuficientes mais relevantes a necessidade de formação docente em inclusão no ES (100\%), as condições de trabalho (50\%), o número de profissionais, os recursos financeiros e o não-envolvimento dos estudantes (37.5\%) (Tabela 4).

\section{DISCUSSÃO}

Considerando as questões levantadas, a investigação revela que existem algumas semelhanças, mas também diferenças no funcionamento dos Serviços de Apoio nos países em análise. Embora as condições nos dois países não sejam idênticas, pode-se considerar que estão sendo 
desenvolvidas as bases para o sucesso e para um projeto de vida socialmente justo. Sem dados sobre estas questões, não é possível saber se as iniciativas estão realmente fazendo uma diferença positiva na vida destes alunos.

Como em outros estudos, o acesso ao ES para pessoas com deficiência apresenta dificuldades, bem como desafios e oportunidades (DATTA; TALUKDAR, 2017; GRIMES; SOUTHGAT; SCEVAK; BUCHANAN, 2019; MESSIOU, 2019; MORIÑA, 2019; STIEFEL; SHIFERAW; SCHWARTZ; GOTTFRIED, 2018; VAN MIEGHEM; VERSCHUEREN; PETRY; STRUYF, 2020).

Embora haja uma evolução significativa, parece que "a expansão não democratizou efetivamente este nível de ensino, que historicamente evidencia marcas de seleção e exclusão" (COSTA; PIECZKOWSKI, 2020, p. 3). A educação inclusiva é um processo contínuo que deve oferecer uma educação de qualidade a todos os alunos, respeitando a diversidade e eliminando todas as formas de discriminação (UNITED NATIONS, 2007); portanto, o ES deve continuar a desenvolver uma ampla gama de serviços para atender às necessidades dos estudantes. Estes podem incluir aconselhamento e tutoria académica especializada, além das acomodações e tecnologia assistida que os Serviços de Apoio fornecem (NOLAN; GLEESON; TREANOR; MADIGAN, 2015).

Quanto às características gerais dos Serviços de Apoio, existem diferenças significativas entre as realidades brasileira e portuguesa, desde as nomenclaturas utilizadas para identificar os serviços até às especificidades relacionadas com a estrutura e organização. Este resultado é corroborado pelas diferentes denominações que encontramos na literatura para esses serviços de apoio (COSTA; PIECZKOWSKI, 2020).

A implementação e o desenvolvimento dos Serviços de Apoio são mais recentes no Brasil do que em Portugal. Estes resultados contrastam com a existência de uma política central definida no Brasil e que não existe em Portugal (MELO; MARTINS, 2016). No que se refere aos recursos financeiros e à autonomia, a realidade é um pouco diferente nos dois países: ao contrário do Brasil, em Portugal, a maioria dos serviços não tem orçamento próprio. Esta é uma questão significativa porque o crescente reconhecimento das funções dos Serviços de Apoio e o número crescente de estudantes com deficiência no ES exige orçamentos mais substanciais e específicos para promover serviços mais eficazes. Este aspecto é particularmente importante, tendo em consideração que os princípios da inclusão precisam ser difundidos e incorporados nas políticas educacionais, pois a deficiência não pode ser conceituada apenas como uma limitação da pessoa, mas esta é fundamentalmente devida às precárias estruturas físicas e sociais (COSTA; PIECZKOWSKI, 2020).

No geral, a acessibilidade aos serviços e espaços internos (ou seja, onde ocorrem os serviços) foi avaliada como positiva. Os resultados indicaram que, em ambos os países, tem havido uma crescente conscientização sobre os serviços que superam as barreiras existentes que dificultam o acesso, a permanência e o sucesso dos alunos com deficiência no ES. No entanto, algumas barreiras permanecem, destacando o desafio contínuo das questões de acessibilidade nas Instituições de Ensino Superior. Estes dados são corroborados em diversas investigações (ACKAH-JNR; DANSO, 2019; FOTIM, 2011; MESSIOU, 2019; MORIÑA, 2019; NDLOVU; WALTON, 2016; PRIYANKA; SAMIA, 2018).

De acordo com FOTIM (2011) os Serviços de Apoio devem fornecer assistência adequada e oportunidades para os estudantes com deficiência para permitir que estes possam competir em igualmente com os seus colegas nos ambientes académicos. Também é importante que estes Serviços de Apoio ajudem os estudantes no acesso a todos os programas, serviços e atividades patrocinadas pelas universidades. Em ambos os países, as Instituições de Ensino Superior designaram um coordenador para estes serviços, uma situação comum em outros países. Neste sentido, Parker (2000) defendeu a necessidade e a importância de um coordenador responsável pelas questões da deficiência nas universidades. Também recomendou o desenvolvimento de um código de ética para promover as melhores práticas.

Em relação aos aspectos demográficos, a maioria dos coordenadores dos Serviços de Apoio são mulheres. A grande maioria possui doutoramento e suas áreas de formação estão relacionadas com as Ciências Sociais e Humanas; têm também formação em Educação Especial e experiência em trabalhar com pessoas com deficiência. Os profissionais que atuam nos Serviços de Apoio são mais numerosos e diversificados no Brasil, variando de administrativos a meio tempo, a serviços constituídos por 
coordenador e alguns funcionários permanentes, além de voluntários para atender os estudantes. Em geral, parece que a especificidade da equipa depende das deficiências dos estudantes. Em Portugal, alguns voluntários apoiam estudantes com deficiências motoras, nomeadamente na deslocação para as salas de aula. Estes dados foram corroborados no estudo desenvolvido pela Foundation of Tertiary Institutions of the Northern Metropolis (FOTIM, 2011).

Também é importante acrescentar as dificuldades apontadas por ambos os países quanto à independência dos serviços na definição de projetos que possam ser relevantes para o sucesso e inclusão dos estudantes com deficiência.

Os resultados indicam que os Serviços de Apoio no Brasil dependem principalmente das faculdades, e os de Portugal dependem principalmente do Reitor/Serviços da Reitoria. No Brasil, os serviços podem dar respostas mais adequadas às reais necessidades do sestudantes atendendo à maior proximidade. Em Portugal, na ausência de uma política definida de forma centralizada, muitos Reitores /Reitorias criaram uma estrutura comum para toda a universidade.

Os dados também indicam que as deficiências mais prevalentes dos estudantes em ambos os países são a deficiência física, a deficiência visual e a deficiência auditiva. Menos prevalentes são as deficiências múltiplas e as deficiências cognitivas. Em Portugal, outras condições são designadas como as doenças psiquiátricas, a dislexia, as doenças crónicas e as doenças neurológicas. Estas resultados são diferentes dos relatadas pelo estudo desenvolvido pelo Departamento de Educação (PEQIS) em que as condições relatadas de deficiência dos estudantes foram 31\% dificuldades de aprendizagem específicas, 18\% ADD/ADHD, 15\% doenças mentais/condições psicológicas ou psiquiátricas e 11\% condições de comprometimento de saúde (RAUE; LEWIS, 2011).

É importante notar que estes dados refletem apenas os estudantes que foram apoiados pelo Serviços de Apoio: muitos estudantes podem ser resistentes e optar por não revelar as suas deficiências por medo de discriminação; o que foi observado em vários estudos (ACKAH-JNR; DANSO, 2019; NDLOVU; WALTON, 2016; SMITH; WOODHEAD; CHIN-NEWMAN, 2019; STRNADOVÁ; HÁJKOVÁ; KVĚTOŇOVÁ, 2015).

Com relação ao funcionamento dos Serviços de Apoio, embora serviços semelhantes sejam oferecidos em ambos os países, argumentamos que os serviços devem ir além do status atual para construir um ambiente inclusivo e implementar tecnologia e dispositivos de assistência para garantir a aprendizagem e metodologias e processos de educação inclusivos (VITALAKI; KOURKOUTAS; HART, 2018). Para uma eficaz agenda de apoios à deficiência, deve-se criar uma maior consciência entre o corpo docente e a equipa em relação às questões da deficiência. Além disso, para garantir a igualdade de oportunidades no Ensino Superior, é fundamental incorporar os princípios da educação inclusiva e do desenho universal para a aprendizagem nas políticas e práticas universitárias (MORIÑA, 2017).

Enquanto no Brasil existe uma maior orientação para as parcerias comunitárias e projetos de desenvolvimento de investigação de apoio a estudantes com deficiência, em Portugal a resposta dos Serviços de Apoios consiste essencialmente em disponibilizar recursos de tecnologia assistida para os estudantes e produção de materiais. Para promover verdadeiros contextos inclusivos e de desenvolvimento devem ser desenvolvidas equipas multidisciplinares.

Significativamente, são necessários quer recursos didáticos (e.g., materiais e tecnologias adaptadas), como profissionais especializados disponíveis para os Serviços de Apoio. As barreiras citadas pelos coordenadores que dificultam a implementação do Desenho Universal incluíram recursos limitados de pessoal especializado para fornecer formação em questões de acessibilidade e os custos associados à aquisição de tecnologia apropriada. Ressalta-se que, também no estudo de Lipka, Baruch e Meer (2019) muitos docentes relataram não ter participado em nenhuma atividade de formação.

\section{NOTAS FINAIS}

Explorar semelhanças e diferenças entre os serviços oferecidos pode promover a compreensão e orientações adicionais para ajudar a reponder às necessidades apresentadas pelos estudantes a nível da sua autonomia, sucesso académico, competências e relacionamento. O objetivo da Universidade é permitir que cada aluno alcance os seus objetivos académicos (QVORTRUP; 
QVORTRUP, 2018). Os Serviços de Apoio devem fornecer programas e serviços destinados a apoiar e incentivar a inclusão dos estudantes com deficiência na comunidade universitária. Estes serviços devem auxiliar na criação de uma comunidade universitária acessível, onde os estudantes com deficiência tenham oportunidades iguais para participar plenamente em todos os aspetos do ambiente educacional. Devem apoiar ainda os ambientes de ensino e aprendizagem por meio de parcerias e formação contínua para estudantes, professores e funcionários.

Os resultados da presente pesquisa revelam que os Serviços de Apoio avaliam e mantêm documentos relacionados com a deficiência, certificam a elegibilidade para os serviços, determinam acomodações razoáveis e desenvolvem planos para a provisão de tais acomodações, auxiliam os alunos na negociação de barreiras relacionadas com a deficiência tendo em vista a sua educação, esforçam-se para melhorar o acesso a programas, atividades e instalações universitárias para os estudantes com deficiência e promover uma maior conscientização sobre as questões da deficiência no campus. Contribuem para a inclusão académica e social dos estudantes com deficiência nos ambientes universitários. É vital que uma diversificada gama de apoios cruciais sejam disponibilizados para os estudantes com deficiência através destes serviços e que cada serviço adote uma abordagem proativa para identificar áreas que promovam e garantam a inclusão e o sucesso académico para todos os alunos (FOSSEY; CHAFFEY; VENVILLE; PRISCILLA; DOUGLAS; BIGBY, 2017; KIMBALL; WELLS; OSTIGUY; MANLY, 2016). Outros estudos observaram que estes serviços poderiam ter maior autonomia e comunicação mais direta com a gestão universitária e que deveriam ter maior participação nas questões da deficiência nas universidades (FOTIM, 2011; NDLOVU; WALTON, 2016). Outro fator importante são as perceções negativas sobre as capacidades dos estudantes e as baixas expetativas em relação ao seu desempenho académico. Face a estas expetativas negativas, alguns estudantes podem optar por não revelar as deficiências invisíveis por medo de serem estigmatizados (MAJOKO; DUNN, 2018; VLACHOU; PAPANANOU, 2018); consequentemente, tais estudantes podem não receber o apoio de que necessitam, prejudicando seu sucesso e carreira académica (ACKAH-JNR; DANSO, 2019).

Segundo Beck, Castillo, Fovet, Mole e Noga (2014), existem várias práticas que os gabinetes de atendimento à deficiência podem implementar para promover o acesso aos serviços. Por exemplo, os estudantes podem ter reuniões virtuais para evitar o medo do estigma. Estes serviços podem desenvolver novas maneiras de alcançar os estudantes que, de outra forma, não procurariam os serviços por conta própria. Para satisfazer as necessidades destes estudantes, poderiam fornecer informações em panfletos em todas as universidades, permitindo que os estudantes consultassem os materiais em local privado, para evitar o estigma. Estes serviços desempenham um papel importante no desenvolvimento de uma compreensão mais complexa da diversidade e inclusão em toda a comunidade universitária. Um objetivo importante é influenciar as perceções dentro da universidade, passando de uma abordagem baseada na limitação e défice para uma nova compreensão, baseada na promoção e desenvolvimento das habilidades dos estudantes.

Neste sentido, argumenta-se que esses serviços de apoio ao estudante podem apoiar os alicerces para mudanças educacionais na "dimensão pedagógica, enraizada em pedagogias críticas, capacitação e educação centrada no aluno, englobando processos educacionais inclusivos e dialógicos para fortalecer a não agência estudantil quer para aprender, quer para reverter as exclusões educacionais e sociais acumuladas" (LAYA, 2020, p. 2).

O processo de inclusão no ES leva tempo e não terá sucesso se os recursos apropriados não estiverem disponíveis (PRIYANKA; SAMIA, 2018). É importante lembrar que as universidades não podem resolver e superar todas as barreiras e obstáculos que enfrentam individualmente. É vital desenvolver uma política abrangente com diretrizes para a educação inclusiva nas universidades, considerando as contribuições de todas as partes (e.g., políticos legisladores, Instituições de Ensino Superior, professores, alunos e comunidade) (ACKAH-JNR; DANSO, 2019; LÓPEZ-GAVIRA; MORIÑA; MORGADO, 2019; MESSIOU, 2019; VITALAKI; KOURKOUTAS; HART, 2018; VLACHOU; PAPANANOU, 2018). Laya (2020) menciona que a dimensão pedagógica da equidade implica mais do que apenas abrir as portas da universidade às populações tradicionalmente excluídas.

Estes serviços beneficiariam de uma formação contínua, de forma a ajudar a promover e a preencher as necessidades psicológicas dos estudantes. A formação e a competência profissional no apoio à deficiência é outro fator que afeta estes estudantes com deficiência e pode levá-los a se 
envolverem com os Serviços de Apoio enquanto frequentam a universidade. Lipka, Baruch e Meer (2019) também observam a importância de fornecer aos professores informações atualizadas sobre as características académicas e cognitivas dos estudantes com deficiência para aumentar a capacidade dos membros do corpo docente para compreender e apoiar estes estudantes.

Assim, considerando o paradigma do Desenho Universal para a Aprendizagem (DUA), as Instituições de Ensino Superior devem adotar uma política que incorpore os conceitos e princípios do Desenho Universal para responder de forma eficaz às necessidades de todos os estudantes (MEYER; ROSE; GORDON, 2014). De acordo com o Centre for Applied Special Technology (CAST) e as considerações de Rose e Meyer, o DUA baseia-se num conjunto de princípios e estratégias que se concentram no ensino, aprendizagem, desenvolvimento de currículo e outros processos relacionados, como a avaliação. O DUA é baseado em processos cerebrais, bem como na pesquisa de tecnologias de informação e comunicação e é projetado para responder às diferenças individuais na aprendizagem. Este novo paradigma postula que o currículo deve incluir alternativas para se tornar mais acessível e apropriado para indivíduos com diferentes origens, estilos de aprendizagem, habilidades e deficiências, em contextos de aprendizagem variados (MEYER; ROSE; GORDON, 2014).

A deficiência pode afetar qualquer pessoa em qualquer fase da vida, tornando-se um aspeto definidor da identidade de alguém. Como Myers, Lindburg e Nied (2014, p. 107) referem: "A deficiência é uma condição humana. Como tal, logicamente faz parte da diversidade.

\section{Implicações, limitações, e investigação futura}

Este trabalho é relevante para garantir que os administradores dos campus se tornem mais sensíveis a ambientes inclusivos de maneira adequada e informada. Esperamos que esta sensibilidade guie as Instituições de Esino Superior e profissionais do Brasil, de Portugal e outros países a desenvolver estratégias significativas para melhor apoiar os estudantes com deficiência nas universidades.

Existem várias limitações neste estudo. Uma limitação importante decorre do objetivo do estudo, que consistia em analisar o nível organizacional, ao invés de examinar áreas-chave na prestação de apoio à deficiência, como divulgação e desenvolvimento de serviços inclusivos. Estudos futuros deveriam tentar recolher uma amostra mais diversa e teria sido particularmente importante incluir professores e os próprios estudantes para fornecer uma análise mais aprofundada. Outras pesquisas combinando as vozes dos alunos e as comunidades académicas em várias Instituições de Ensino Superior permitiriam uma exploração mais profunda das considerações levantadas neste artigo. Uma outra limitação pode envolver algum viés de respostas. Embora as perguntas feitas durante a pesquisa incluíssem uma mistura de perguntas de escolha fechada e forçada, escalas Likert e oportunidades para comentar, devido à natureza sensível do assunto, é possível que alguns participantes estivessem menos abertos para discutir as suas experiências do que outros.

Os resultados deste estudo fornecem uma visão sobre o papel dos serviços de apoio universitário para atender às necessidades dos estudantes com deficiência. No entanto, mais pesquisas ainda são necessárias para melhor atender os estudantes universitários com deficiência e entender a sua motivação para se autodeclararem e utilizarem os serviços de apoio universitário (MAJOKO; DUNN, 2018; LÓPEZ-GAVIRA; MORIÑA; MORGADO, 2019).

É importante que as universidades adotem uma abordagem inclusiva. Este estudo pode contribuir para a disseminação de práticas que possam ajudar as universidades a adotarem o paradigma do Desenho Universal de Aprendizagem, respondendo de forma mais eficaz às necessidades de todos os estudantes. Segundo Domingo, Pérez-García e Domingo (2019), o construto da inclusão educacional e social é complexo e deve ir além da simples prestação de serviços e estruturas de apoio. A inclusão deve ir além do domínio discursivo, para garantir os direitos de todos os alunos.

Agradecimentos: Os autores gostariam de agradecer a todos os sujeitos que participaram nesta investigação.

Financiamento: Este trabalho foi financiado por fundos nacionais da FCT - Fundação para a Ciência e Tecnologia, I.P., no âmbito do projeto UIDB / 04312/2020; e pela Fundação CAPES - Coordenação 
de Aperfeiçoamento de Pessoal de Nível Superior, Ministério da Educação do Brasil, sob o projeto P99999.002554 / 2015-01.

Declaração de divulgação: Nenhum conflito de interesse potencial foi relatado pelos autores.

\section{REFERENCES}

ACKAH-JNR, Francis; DANSO, Joyce. Examining the physical environment of Ghanaian inclusive schools: how accessible, suitable and appropriate is such environment for inclusive education? International Journal of Inclusive Education, v. 23, n. 2, p. 188- 208, 2019. Available in: https://doi.org/10.1080/13603116.2018.1427808. Access in: 04 jul. 2020.

BECK, Tanja; CASTILLO, Patricia Diaz; FOVET, Frederic; MOLE, Heather; NOGA, Brodie. Applying Universal Design to Disability Service Provision: Outcome Analysis of a Universal Design. (UD). Audit. Journal of Postsecondary Education and Disability, v. 27, n. 2, p. 209-222, 2014. Available in: https://files.eric.ed.gov/fulltext/EJ1040544.pdf. Access in: 03 jul. 2020.

COSTA, Joana Maria de Morales; PIECZKOWSKI, Tania Mara Zancanaro. Inclusion of students with disabilities in higher education from the perspective of university management. Educação em Revista, Belo Horizonte, v. 36, 2020. Available in: http://www.scielo.br/scielo.php?script=sci_arttext\&=S010246982020000100209\&lng=en\&nrm=iso. Access in: 16 oct. 2020.

DATTA, Poulommee; TALUKDAR, Joy. The impact of support services on students' test anxiety and/or their ability to submit assignments: a focus on vision impairment and intellectual disability. International Journal of Inclusive Education, v. 21, n. 2, p. 160-171, 2017. Available in: https://doi.org/10.1080/13603116.2016.1193561. Access in: 16 oct. 2020.

FOSSEY, Ellie; CHAFFEY, Lisa; VENVILLE, Annie; PRISCILLA, Ennals; DOUGLAS, Jacinta; BIGBY, Christine. Navigating the complexity of disability support in tertiary education: perspectives of students and disability service staff. International Journal of Inclusive Education, v. 21, n. 8, p. 822832, 2017. DOI: 10.1080/13603116.2017.1278798.

FOTIM. Disability in Higher Education: Project Report. 2011. Available in: http://www.students.uct.ac.za/usr/disability/reports/annual_report_10_11.pdf. Access in: 06 jul. 2020.

GRIMES, Susan; SOUTHGAT, Erica; SCEVAK, Jill; BUCHANAN, Rachel. University student perspectives on institutional non-disclosure of disability and learning challenges: reasons for staying invisible. International Journal of Inclusive Education, v. 23, n. 6, p. 639-655, 2019. DOI: 10.1080/13603116.2018.1442507.

HOUSE OF COMMONS LIBRARY. People with disabilities in employment. 2020. Available in: https://researchbriefings.parliament.uk/ResearchBriefing/Summary/CBP-7540. Access in: 06 nov. 2020.

KIMBALL, Ezekiel; WELLS, Ryan; OSTIGUY, Catherine; MANLY, Alexandra Lauterbach. Students with Disabilities in Higher Education: A Review of the Literature and an Agenda for Future Research. In.: MICHAEL, Paulsen (Ed.). Higher Education: Handbook of Theory and Research. Switzerland: Springer International Publishing, 2016. (HATR, v. 31, p. 91-156). DOI: 10.1007/978-3-319-26829-3_3. 
LAYA, Marisol Silva. La dimensión pedagógica de la equidad en educación superior. Archivos Analíticos de Políticas Educativas, v. 28, n. 1, 2020. Available in: https://dialnet.unirioja.es/servlet/articulo?codigo=7444004. Access in: 06 nov. 2020.

LIPKA, Orly; BARUCH, Alona Forkosh; MEER, Yael. Academic support model for post-secondary school students with learning disabilities: student and instructor perceptions. International Journal of Inclusive Education, v. 23, n. 2, p. 142-157, 2019. DOI: 10.1080/13603116.2018.1427151.

LIPKA, Orly; KHOURI, Marlyn; SHECTER-LERNER, Michal. University faculty attitudes and knowledge about learning disabilities. Higher Education Research \& Development, v. 39, n. 5, 2020. DOI: $10.1080 / 07294360.2019 .1695750$.

LÓPEZ-GAVIRA, Rosario; MORIÑA, Anabel; MORGADO, Beatriz. Challenges to inclusive education at the University: the perspective of students and disability support service staff. Innovation: The European Journal of Social Science Research, v. 34, n. 3, p. 292-304, 2019. DOI: https://doi.org/10.1080/13511610.2019.1578198.

MARTINS, Maria Helena; BORGES, Maria Leonor; FONSECA, Henrique; GONÇALVES, Teresa; FERREIRA, Joana. (2015). Estudantes não-tradicionais no Ensino Superior: Investigar para guiar a mudança institucional. Relatório apresentado à Fundação para a Ciência e Tecnologia no âmbito do projeto PTDC/IVC-PEC/4886/2012. [Non-traditional students in Higher Education: Investigate to guide institutional change. Report presented to the Foundation for Science and Technology within the scope of the PTDC / IVC-PEC / 4886/2012 project]. Faro: Universidade do Algarve.

MAJOKO, Tawanda; DUNN, Michael William (Reviewing editor). Participation in higher education: Voices of students with disabilities. Cogent Education, v. 5, n. 1, 2018. Available in: https://doi.org/10.1080/2331186X.2018.1542761. Access in: 06 mai. 2020.

MARTOS, Lorena Domingo; PÉREZ-GARCÍA, Purificación; SEGOVIA, Jesus Domingo. Miradas críticas de los profesionales de la educación ante las respuestas educativas al reto de la inclusión en la escuela andaluza. Archivos Analíticos de Políticas Educativas, v. 27, n. 118, 2019. Available in: https://doi.org/10.14507/epaa.27.4185. Access in: 03 jul. 2020.

MELO, Ricardo Lins Vieira; MARTINS, Maria Helena. Legislação para estudantes com deficiência no ensino superior no Brasil e em Portugal: algumas reflexões. [Legislation for students with disabilities in higher education in Brazil and Portugal: some reflections]. Acta Scientiarum. Education, v. 38, n. 3, p. 259-269, 2016. DOI: 10.4025/actascieduc.v38i3.30491.

MESSIOU, Kyriaki. Collaborative action research: facilitating inclusion in schools. Educational Action Research, v. 27, n. 2, p. 197-209, 2019. Available in: https://doi.org/10.1080/09650792.2018.1436081. Access in: 02 jul. 2020.

MEYER, Anne; ROSE, David; GORDON, David. Universal design for learning: Theory and practice. Wakefielf, MA: CAST Professional Publishing, 2014.

MORIÑA, Anabel. Inclusive education in higher education: challenges and opportunities. European Journal of Special Needs Education, v. 32, n. 1, p. 3-17, 2017. DOI: 10.1080/08856257.2016.1254964.

MORIÑA, Anabel. Learning from experience: training for faculty members on disability. Perspectives: Policy and Practice in Higher Education, v. 23, n. 2-3, p. 86-92, 2019. DOI: 10.1080/13603108.2018.1534759. 
MORIÑA, Anabel; MORGADO, Beatriz; LÓPEZ-GAVIRA, Rosario. How do disability support offices contribute to inclusive education in the university. Disability and Society, 2017, v. 32, n. 10, p. 1608-1626, 2017. DOI:10.1080/09687599.2017.1361812.

MYERS, Karen; LINDBURG, Jaci Jenkins; NIED, Danielle. Allies for Inclusion: Disability and Equity in Higher Education. United States: Jossey-Bass, 2014. (Association for the Study of Higher Education (ASHE) Report, v. 39, n. 5).

NDLOVU, Sibonokuhle; WALTON, Elizabeth. Preparation of students with disabilities to graduate into professions in the South African context of higher learning: Obstacles and opportunities. African Journal of Disability, v. 5, n. 1, 2016. Available in: http://www.ajod.org/index.php/ajod/article/view/150. Access in: 01 jul. 2020.

NOLAN, Clodagh; GLEESON, Claire; TREANOR, Declan; MADIGAN, Susan. Higher education students registered with disability services and practice educators: issues and concerns for professional placements. International Journal of Inclusive Education, v. 19, n. 5, p. 487-502, 2015. DOI: 10.1080/13603116.2014.943306.

PARKER, Viv. Developing a code of practice for disability coordinators. European Journal of Special Needs Education, v. 15, n. 3, p. 275-284, 2000. DOI: 10.1080/088562500750017880.

PRIYANKA, Sharma; SAMIA, Kohli. Barriers to inclusive education for children with special needs in schools of Jammu. The International Journal of Indian Psychology, v. 6, n. 1, p. 93-105, 2018. Available in: https://doi.org/10.25215/0601.012. Access in: 02 jul. 2020.

QVORTRUP, Ane; QVORTRUP, Lars. Inclusion: Dimensions of inclusion in education. International Journal of Inclusive Education, v. 22, n. 7, p. 803- 817, 2018. Available in: https://doi.org/10.1080/13603116.2017.1412506. Access: 05 jul. 2020.

RAUE, Kimberley; LEWIS, Laurie. Students with Disabilities at Degree-Granting Postsecondary Institutions. Washington: U.S. Department of Education, National Center for Education Statistics, 2011. Available in: https://nces.ed.gov/pubs2011/2011018.pdf. Access in: 06 jul. 2020.

SMITH, Sara; WOODHEAD, Erin; CHIN-NEWMAN, Christina. Disclosing accommmodation needs: exploring experiences of higher education students with disabilities. International Journal of Inclusive Education, v. 25, n. 12, 1358-1374, 2019. DOI: 0.1080/13603116.2019.1610087.

STIEFEL, Leanna; SHIFERAW, Menbere; SCHWARTZ, Amy Ellen; GOTTFRIED, Michael. Who feels included in school? Examining feelings of inclusion among students with disabilities. Educational Researcher, v. 47, n. 2, p. 105-120, 2018. Available in: https://doi.org/10.3102/0013189X17738761. Access in: 02 jul. 2020.

STRNADOVÁ, Iva; HÁJKOVÁ, Vanda; KVĚTOŇOVÁ, Lea. Voices of university students with disabilities: inclusive education on the tertiary level - a reality or a distant dream?, International Journal of Inclusive Education, v. 19, n. 10, p. 1080-1095, 2015. DOI: 10.1080/13603116.2015.1037868.

TITCHKOSKY, Tanya. To Pee or Not to Pee? Ordinary Talk about Extraordinary Exclusions in a University Environment. Canadian Journal of Sociology, v. 33, n. 1, p. 37-60, 2008. Available in: https://journals.library.ualberta.ca/cjs/index.php/CJS/article/view/1526/1058. Access in: 02 jul. 2020.

UNESCO. A guide for ensuring inclusion and equity in education. The Global Education 2030 Agenda. Geneva, 2017. Available in: http://unesdoc.unesco.org/images/0024/002482/248254e.pdf. Access in: 01 jul. 2020. 
UNITED NATIONS. Convention on the Rights of Persons with Disabilities. Geneva, 2006. Available in: https://www.un.org/development/desa/disabilities/convention-on-the-rights-ofpersonswith-disabilities.html. Access in: 02 jul. 2020.

UNITED NATIONS. Disabilities, From Exclusion to Equality, Realizing the rights of persons with disabilities. Geneva, 2007. (Handbook for Parliamentarians on the Convention on the Rights of Persons with Disabilities and its Optional Protocol, 14). Available in: http://www.un.org/disabilities/documents/toolaction/ipuhb.pdf. Access in: 02 jul. 2020.

UNITED NATIONS. System Task Team on the post 2015 UN Development Agenda: Realizing the future we want for all. 2015. Available in: http://www.un.org/millenniumgoals/pdf/Post_2015_UNTTreport.pdf. Access in: 02 jul. 2020.

VAN MIEGHEM, Aster; VERSCHUEREN, Karine; PETRY, Katja; STRUYF, Elke. An analysis of research on inclusive education: a systematic search and meta review. International Journal of Inclusive Education, v. 24, n. 6, p. 1-15, 2020. Available in: https://doi.org/10.1080/13603116.2018.1482012. Access in: 04 jul. 2020.

VITALAKI, Elena, KOURKOUTAS, Elias; HART, Angie. Building inclusion and resilience in students with and without SEN through the implementation of narrative speech, role play and creative writing in the mainstream classroom of primary education. International Journal of Inclusive Education, v. 22, n. 12, p. 1306-1319, 2018. Available in: https://doi.org/10.1080/13603116.2018.1427150. Access in: 02 jul. 2020.

VLACHOU, Anastasia; PAPANANOU, Ioanna. Experiences and perspectives of Greek higher education students with disabilities. Educational Research, v. 60, n. 2, p. 206-221, 2018. DOI: 10.1080/00131881.2018.1453752.

WILLIAMS, Matthew; POLLARD, Emma; TAKALA, Helena; HOUGHTON, Ann-Marie. Review of Support for Disabled Students in Higher Education in England, Report to the Office for Students by the Institute for Employment Studies and Researching Equity, Access and Participation. Brighton: Institute for Employment Studies, 2019. Available in: https://www.officeforstudents.org.uk/media/a8152716-870b-47f2-8045-fc30e8e599e5/review-ofsupport-for-disabled-students-in-higher-education-in-england.pdf. Access in: 02 jul. $2020 .$.

Submissão: 08/01/2021

Aprovado: 05/08/2021 